\title{
Study of the interaction of the rupture zones of contiguous anchor plates in analogical medium
}

\author{
H. Abbad ${ }^{1}$, N. Elmeiche ${ }^{12}$, I. Mechab ${ }^{23}$ and C. Zemri ${ }^{21}$ \\ ${ }^{1}$ Université Djillali Liabes, LGCE, Département de Génie Civil et Travaux Publics, Sidi Bel Abbes, Algérie \\ ${ }^{2}$ Université Mustapha Stambouli, Département de Génie Civil, Mascara, Algérie \\ ${ }^{3}$ Université Djillali Liabes, LMPM, Département de Génie Mécanique, Sidi Bel Abbes, Algérie
}

\begin{abstract}
Knowing what occurs above anchor plates is certainly instructive. In this general vision of the interaction soil-anchor plates that our work was directed. An experimental study which required the design and implantation of a model containing plastic granules powder to similate a natural environment, is presented. The latter is subjected to the removal of anchor paltes. For each test, digital photographs are taken to materialize different deformed configurations during the pullout process. These photos processed in couples by the 7D software (image correlation) giving the evolution of the displacement field and plane strain analogical environment. Particular attention is paid to the discussion of the interference of rupture zones of contiguous anchors by reducing the distance between plates.
\end{abstract}

\section{Introduction}

Les plaques d'ancrage sont des éléments structuraux légers employés pour résister à des forces d'arrachement «traction» sollicitant les pylônes, les mâts de transmission et les structures soumises à la poussée d'Archimède telle que les stations offshore. Des études théoriques 'Meyerhof [1]; Kumar et Kouser [2]', et expérimentales sur modèle réduits sur des plaques contigües ancrées dans un sol pulvérulent effectuées par 'Hanna et Sparks [3] ; Geddes et Murray [4]' ont mis en évidence l'influence du sol d'ancrage, de la géométrie des plaques, de la profondeur de d'ancrage et de la distance entre deux plaques voisines sur leur comportement. Voir de plus près ce qui se passe dans le milieu environnant deux plaques d'ancrage voisines, reste cependant toujours instructif. Cela est d'autant plus vrai que le comportement du milieu est conditionné par l'existence des configurations particulières des plaques 'Abbad [5]'. L'analyse cinématique de ces milieux, moyennant une modélisation physique, permet de se rendre compte du comportement réel du milieu étudié et surtout répondre à des interrogations des problématiques précises. L'utilisation d'un milieu analogique dans ce genre de modélisation rapproche à la compréhension de la phénoménologie des sols réels tant que les deux matériaux ont des points communs tels que l'incompressibilité des grains et leur caractère frottant.

\section{Modèle expérimental}

Le modèle réduit sur lequel nous avons réalisé différents essais est un dispositif parallélépipédique, de dimensions égales à $(100 \times 80 \times 5) \mathrm{cm}$, conçu pour contenir des granulés en plastique (matériau analogique) simulant un milieu granulaire. La paroi d'avant du modèle est constituée d'une plaque de verre de $6 \mathrm{~mm}$ d'épaisseur, avec un indice réfraction constant sur toute sa surface. Les autres parois sont réalisées avec des plaques en bois rigides. Des parallélépipèdes en bois lissé de dimensions $(5 \times 5 \times 1,5) \mathrm{cm}$, modélisant des plaques d'ancrage, sont en contact ponctuel avec des tiges filetée. Des écrous sont soudés à un système d'attache au châssis du modèle, ce qui permet de contrôler l'arrachement des deux plaques en dévissant les tiges. Un déplacement strictement vertical est imposé aux plaques d'ancrage du fait que ces dernières sont guidées par des rails de $5 \mathrm{~mm}$ d'épaisseur, placés sur la paroi arrière, empêchant toute rotation (figure 1).

Les grains en plastique, ronds de diamètre moyen $1 \mathrm{~mm}$, de différentes couleurs (rouge, bleue, jaune, verte et noire) sont mélangés pour matérialiser un milieu visuellement contrasté. Afin d'obtenir un milieu compact et homogène, le matériau est déposé uniformément sur toute la largeur du bac par petites couches de $2 \mathrm{~cm}$ d'épaisseur, chaque couche est compactée moyennant une dame de compactage métallique. Le milieu à un indice des vides de 0,321 , correspondant à une densité relative $\operatorname{Dr}=96 \%\left(\mathrm{e}_{\min }=0.302, \mathrm{e}_{\max }=0.855\right)$. Vu les très faibles contraintes mises en jeu, les grains en plastique 
constituant le milieu étudié sont considérés indéformables.

Afin de décrire le champ de déplacement et de déformation au dessus d'une plaque d'ancrage, plusieurs séries d'essais ont été réalisées. Pour chaque essai réalisé, une série de photos numériques, de résolution $3200 \mathrm{x}$ 2400 pixels, est prise de face, l'axe de la prise de vue étant perpendiculaire au plan du modèle réduit. Ces photos matérialisent différentes configurations déformées du milieu granulaire, engendrées par l'arrachement des plaques d'ancrage. Les images enregistrées sont traitées ultérieurement pour restituer le champ de déplacement pour déterminer le champ de déformation par le logiciel 7D (Vacher et al [6]). Il s'agit d'une méthode de corrélation entre deux images, ces dernières seront comparées en utilisant le coefficient de corrélation du niveau de gris. En d'autres termes, les points de l'image initiale sont retrouvés sur les images successives avec une incertitude de mesure de l'ordre de 0,1pixel, voire 0,01 pixel en petite déformation. L'image pour laquelle la déformation sera analysée est divisée en un certain nombre d'éléments de grille, carrés de côtés 20 pixels.

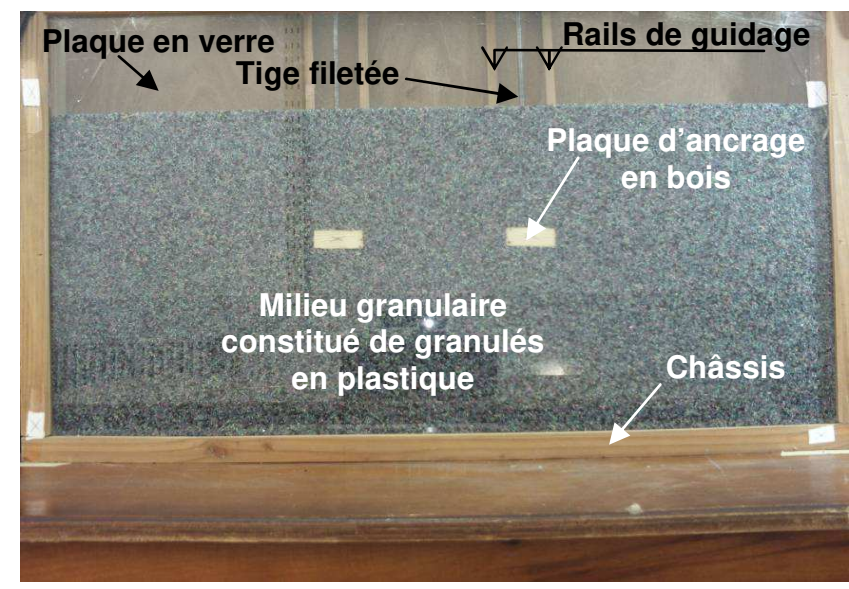

Fig. 1. Vue du modèle réduit.

\section{Déplacements et déformations au- dessus des plaques d'ancrage}

La mise en évidence de l'influence du ratio d'ancrage (H/B) sur le comportement du milieu granulaire audessus des plaques d'ancrage est volontairement cinématique, faisant abstraction de toute mesure de l'état de contrainte. Les essais en question ont été effectués à des profondeurs d'ancrage de la plaque carrée de $\mathrm{H}=3 \mathrm{~B}$, $\mathrm{H}=5 \mathrm{~B}$ et $\mathrm{H}=7 \mathrm{~B}, \mathrm{H}$ et $\mathrm{B}$ étant respectivement la profondeur d'ancrage de la plaque et la largeur de cellesci.

Lors des traitements d'images, un domaine d'étude est défini par l'opérateur. On suivra à volonté l'évolution des déplacements et des déformations dans ce domaine. L'arrachement ou soulèvement imposé à la plaque d'ancrage se fait à des pas constants de l'ordre de 0,266 mm correspondant à 1 pixel.

\subsection{Champ de déplacement}

Les figures $(2 \mathrm{a}, 2 \mathrm{~b}$ et $2 \mathrm{c})$ reproduisent graphiquement les trajectoires des grains pour un arrachement de 20 pixels et pour des distances entre nus des plaques d'ancrage respectives de (3B), (4B) et (6B). On remarque que le déplacement des grains s'accentue verticalement suivant l'axe de la plaque, et horizontalement vers les bords de celle-ci. Les vecteurs de déplacement sont tracés avec un coefficient d'amplification de 7 (700\%). En premier lieu, nous constatons que les vecteurs de déplacement sont globalement verticaux et orientés vers le haut, les trajectoires des grains s'amortissent, à peu près, à l'approche d'un plan horizontal parallèle à la surface libre du massif, suivant un arc incurvé vers le haut.
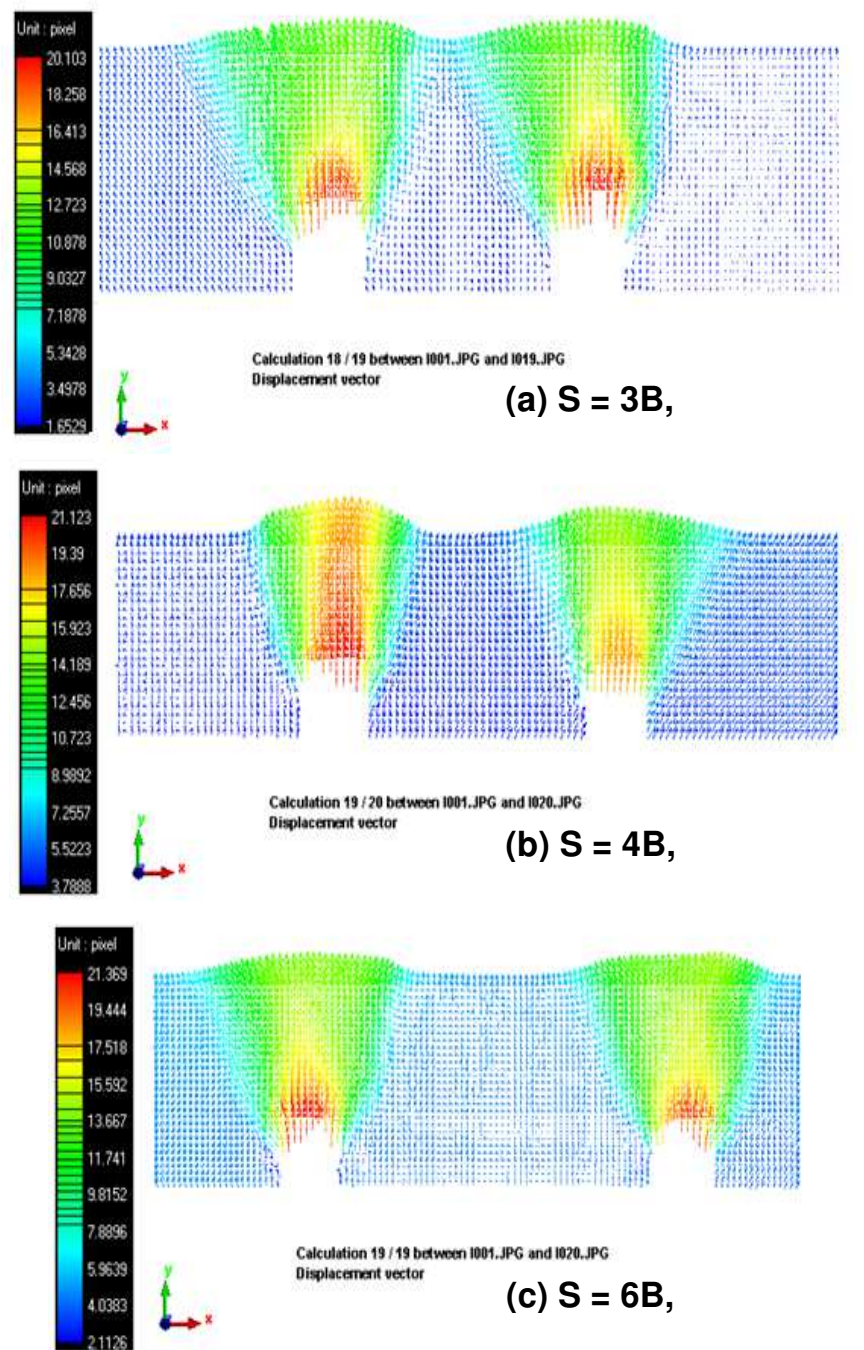

Fig. 2. Vecteurs de déplacements pour différentes distances entre nus de plaques.

Les figures (3a, $3 b$ et $3 c$ ) représentent les champs de déplacement verticaux du matériau soumis à l'arrachement des deux plaques d'ancrage écartées entre nus respectivement de (3B), (4B) et 6B, l'enfoncement est de 20 pixels. Localement, au-dessus de chaque plaque, le milieu se comporte de manière similaire à une plaque isolée. Remarquons que la formation d'un «coin 
rigide » au-dessus chaque plaque confirme la compacité élevée du matériau.

En fonction de l'intensité de l'arrachement des plaques, un fort gradient de déplacement se met en place sur un contour triangulaire légèrement incurvé confirmant l'existence des coins rigides au-dessus des plaques.

On remarque qu'au-delà d'un écartement $\mathrm{S}=4 \mathrm{~B}$ les plaques agissent indépendamment sur le matériau. A une distance $\mathrm{S}=3 \mathrm{~B}$, l'interaction des zones de rupture de chaque plaque crée un bulbe de déplacement vertical qui atteint la valeur de 5.17 pixels au niveau de la surface du massif à mi distance entre nus de plaques.

Le refoulement des grains est limité par des droites inclinées passantes par les nus des plaques, l'inclinaison de ces droites est d'environ $31^{\circ}$.

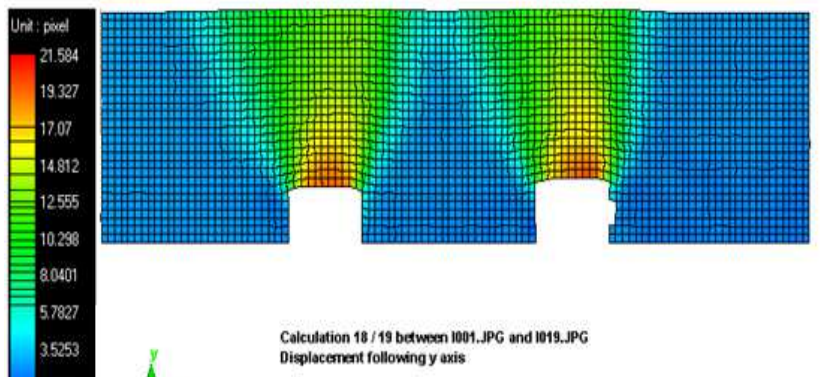

(a) $\mathrm{S}=3 \mathrm{~B}$

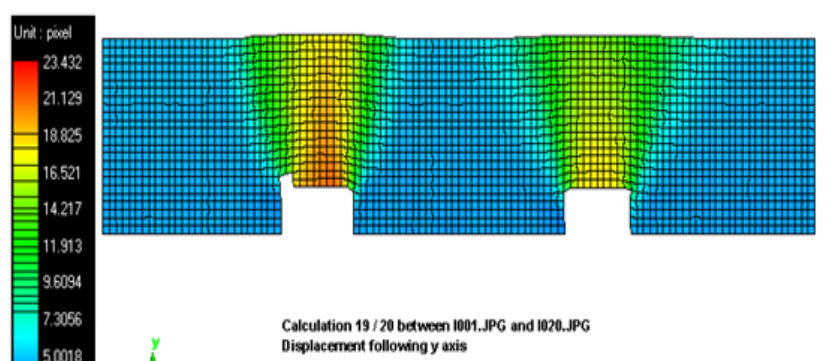

(b) $S=4 B$

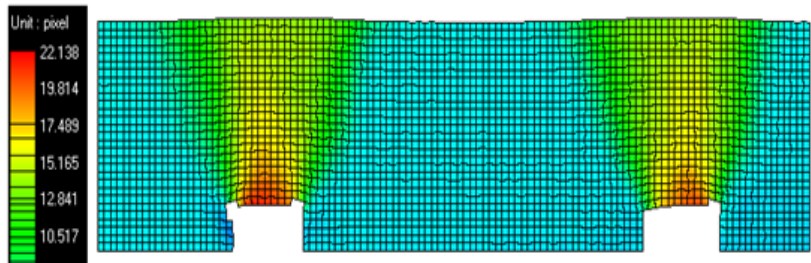

1924

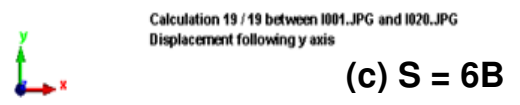

Fig. 3. Champ de déplacement vertical pour différentes distances entre nus de plaques.

La figure 4 schématise la superposition entre les isovaleurs du déplacement vertical pour un arrachement de la plaque de 20 pixels et le mode de rupture préconisée par Ilamparuthi et al en 2002 [7], nous constatons une similitude de forme entre les isovaleurs de déplacement et le mode de rupture généralisé pour un ancrage de $\mathrm{H}=3 \mathrm{~B}$, en effet ce mode de rupture concerne les milieux granulaires denses soumis à l'arrachement d'une plaque d'ancrage superficielle.

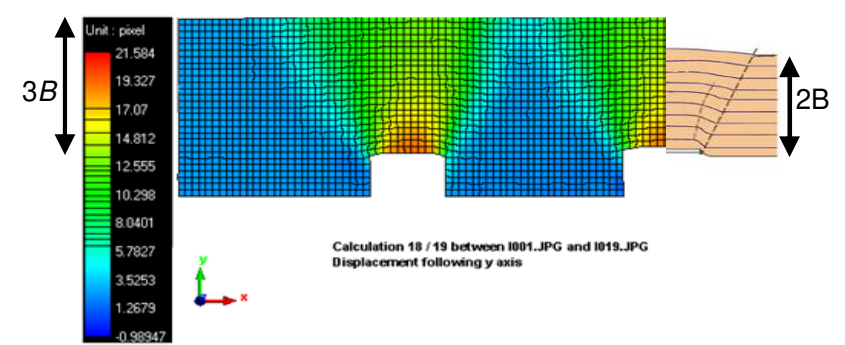

Fig. 4. Superposition entre les isovaleurs du déplacement vertical et les résultats de Ilamparuthi et al. 2002

\subsection{Champ de déformation}

Les figures $(5 \mathrm{a}, 5 \mathrm{~b}$ et $5 \mathrm{c})$ représentent les champs de déformation (cisaillement maximal) dans le milieu granulaire pour un arrachement de 20 pixels et pour des écartements entre nus de plaques respectifs de (3B), (4B) et (6B). On remarque que les bulbes de cisaillement au niveau de la surface du massif à mi distance entre nus de plaques sont d'autant plus grands que les plaques se rapprochent l'une de l'autre avec une plus grande intensité de cisaillement qui atteint la valeur de $3 \%$ pour un écartement de $3 \mathrm{~B}$ alors qu'elle ne dépasse pas les $0.76 \%$ pour un écartement de $4 \mathrm{~B}$ et les $0.38 \%$ pour un écartement de 6B.
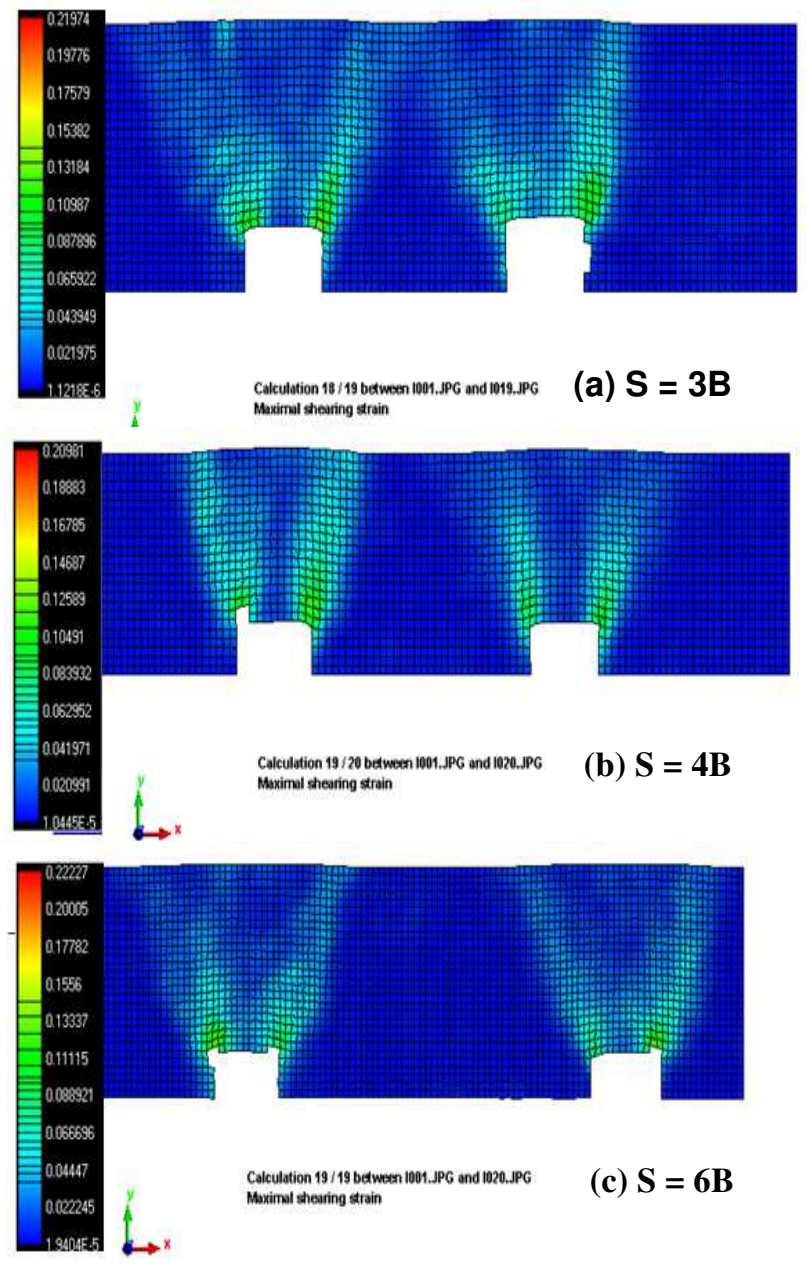

Fig. 5. Champ de déformation (cisaillement maximal) pour différentes distances entre nus de plaques. 


\section{Conclusion}

L'étude expérimentale que nous avons menée, s'est basée principalement sur l'évolution du milieu granulaire soumis à l'arrachement des plaques d'ancrage.

Les essais d'arrachement effectués ont permis de quantifier le champ de déplacement vertical et celui des déformations du milieu granulaire au-dessus des « plaques d'ancrage » grâce à la technique de corrélation d'image. Le choix d'un arrachement de 20 pixels (déplacement imposé) se justifie par le souci de présenter des champs de déformations correspondant à un état de rupture. Une multitude de couple de photos ont été traité, allant jusqu'à un arrachement de 33 pixels et plus. Audelà de 18 pixels, la configuration déformée est pratiquement inchangée, ce qui montre qu'à ce stade de sollicitation, le milieu a atteint sa rupture et qu'un état de plasticité s'installe.

L'interaction des zones de ruptures des plaques voisines est étudiée cinématiquement en faisant varier le paramètre distance entre nus de plaques. Il s'avère qu'un écartement minimal d'environ quatre fois la largeur de la semelle $(4 B)$ est nécessaire pour que deux plaques d'ancrage voisines agissent indépendamment sur le milieu.

La corrélation entre la phénoménologie de l'évolution des champs de déplacement et de déformation au-dessus de la plaque d'ancrage et le mode de rupture du massif d'ancrage, faisant objet d'études antérieures a été mise en évidence grâce à la superposition entre les isovaleurs du déplacement vertical (pour un enfoncement de 20 pixels) et le mode de rupture généralisé défini par Ilamparuthi et al. (2002) [7].

\section{Références}

1. G.C Meyerhof, S.I. Adams, The ultimate uplift capacity of foundations, Can Geotech J 4, 225-244 (1968)

2. J Kumar, K.M Krouzer, Interference effect on the vertical uplift capacity of two shallow horizontal anchors, Géotechnique, 58 10, 821-824 (2008)

3. T.H Hanna, R Sparks, M Yilmaz, Anchor behaviour in sand, J Soil Mech Found Div, ASCE 9811 pp1187 (2007)

4. J.D Geddes, E.J Murray, Plate anchor groups pulled vertically in sand, J Geotech Eng, ASCE, 122 7, 509516 (1996)

5. H Abbad, Mise au point d'un dispositif d'analyse des déformations planes par corrélation d'images, Thèse de doctorat, Université de Sidi Bel Abbès, Algérie (2011)

6. P Vacher, S Dumoulin, Morestin and SMguilTouchal, Bidimensional strain measurement using digital images, Poc. Instn. Mech. Engrs, 213 811-817 (1999)

7. Ilamparuthi $\mathrm{K}$, Dickin EA, Muthukrishnaiah $\mathrm{K}$, Experimental investigation of the uplift capacity of circular plate anchors in sand, Can Geotech J 39, 644-648 (2002) 